\title{
A Cluster Randomized Controlled Trial of Nonpharmacological Interventions for Old-Old Subjects with a Clinical Dementia Rating of 0.5: The Kurihara Project
}

\author{
Masahiro Nakatsuka ${ }^{a}$ Kei Nakamura ${ }^{a}$ Ryo Hamanosono ${ }^{a}$ \\ Yumi Takahashi ${ }^{a}$ Mari Kasai $^{a}$ Yuko Sato ${ }^{a}$ Teiko Suto $^{a}$ \\ Ryoichi Nagatomi $^{b}$ Kenichi Meguro ${ }^{a}$ \\ a Division of Geriatric Behavioral Neurology, Cyclotron and Radioisotope Center (CYRIC), \\ Tohoku University, and ${ }^{b}$ Department of Medicine and Science in Sports and Exercise, \\ Tohoku University School of Medicine, Sendai, Japan
}

Key Words

Nonpharmacological intervention · Old-old subjects · Clinical Dementia Rating

\section{Abstract}

Background: Evidence as to the benefits of nonpharmacological interventions for the boundary state between normal aging and dementia [mild cognitive impairment or a Clinical Dementia Rating (CDR) of 0.5] remains weak due to a lack of positive controls. Aims: To directly compare the effects of cognitive interventions (CI), physical activities (PA) and a group reminiscence approach (GRA), we conducted a pilot study on the basis of a cluster randomized controlled trial design. Method: A total of 127 participants aged $>74$ years with a CDR of 0.5 were cluster randomized into three groups for CI, PA and GRA. The intervention lasted 12 weeks and consisted of weekly group sessions and home assignments. Mini-Mental State Examination (MMSE), Trail Making Test part A (TMT-A), word fluency (WF), 6-meter walk time and Quality of Life (QOL) Face Scale scores were evaluated as primary outcomes. Results: Methodology-related benefits of CI and PA were found for MMSE scores and walk time, respectively. TMT-A, WF and QOL Face Scale scores improved irrespective of the methodologies used. Conclusions: Our findings suggest that $\mathrm{CI}$ and PA may be beneficial to cognitive and physical abilities, respectively. Executive functions and QOL may improve irrespective of the intervention methodologies used. 


\section{Introduction}

The boundary or transitional state between normal aging and dementia, which is defined in various ways such as mild cognitive impairment (MCI) or a Clinical Dementia Rating (CDR) of 0.5 , is recognized as a state of being at high risk of dementia $[1,2]$. Although it is a serious challenge to control the risk of dementia in these people, pharmacological interventions remain unsuccessful [3]. Meanwhile, recent studies have suggested potential benefits of nonpharmacological interventions [3-10]. Among a variety of nonpharmacological methodologies, most popular and potentially beneficial interventions to date include cognitive interventions (CI), physical activities (PA) and a group reminiscence approach with reality orientation (GRA).

CI refer to stimulation, rehabilitation or training of higher cerebral functions $[11,12]$. While many studies have successfully shown a statistically significant improvement in objective and/or subjective measures of cognitive function in people at high risk of dementia $[5,7]$, cognitive exercises may lead to greater benefits than memory strategies on memory [5]. With regard to PA, there are studies suggesting that aerobic [4] as well as resistance [9] exercises may have positive effects on the executive functions of people with MCI. The GRA was introduced in the 1980s and is one of the most popular psychosocial interventions in dementia care. It is based on evocation and discussion with another person or a group about past activities, events and experiences, using a variety of supporting materials [13, 14]. Although the currently available evidence is limited, there are studies suggesting that the GRA may have a favorable effect on the psychosocial well-being [8] and autobiographical memory of demented patients [15]. Meanwhile, the effectiveness for people in the transitional state between normal aging and dementia remains unclear.

All in all, these three popular interventions may be promising candidate methodologies. However, evidence about their specific benefits remains to be gathered, since most of the previous trials lack active controls or direct comparisons between different methodologies. The aim of the present study was to clarify the cognitive, physical and psychological shortterm effects of these three popular nonpharmacological interventions, directly comparing them on the basis of a cluster randomized controlled trial (RCT) design. To our knowledge, this is the first RCT of the GRA in subjects with a CDR of 0.5 and the first trial of nonpharmacological interventions with active controls.

\section{Subjects and Methods}

\section{Subjects}

As part of the community-based project on stroke and dementia prevention in Kurihara City, northern Japan (Kurihara Project) [16], 295 community-dwelling people aged $\geq 75$ years and evaluated as having a CDR of 0.5 [17] were enrolled. The sample size was determined so that the error ranges of the Mini-Mental State Examination (MMSE) [18] scores of the three intervention groups (mentioned below) be within 1 point (95\% confidence interval) according to the calculation based on the data on participants assessed as having a CDR of 0.5 in the Kurihara Project [16]. Following the scoring rule of the CDR [17], the evaluation of each subject was based on consensus achieved via discussions at a clinical conference of medical doctors and public health nurses who saw the participant and his/her family.

All subjects underwent medical checkups including head MRI and blood testing. Blood testing included screenings for syphilis infection and dysfunctions of the thyroid, liver and kidney. Those who were diagnosed with neurodegenerative diseases such as Parkinson's disease, progressive supranuclear palsy or spinocerebellar degeneration were excluded from 
Nakatsuka et al.: A Cluster Randomized Controlled Trial of Nonpharmacological Interventions for Old-Old Subjects with a CDR of 0.5: The Kurihara Project

the study. All diagnoses were made by board-certified neurologists of the Japanese Society of Neurology (Tokyo, Japan). Subjects who had a history of depression or schizophrenia were excluded. While none of the participants used antidepressants or antipsychotic agents, the use of sleeping drugs was not excluded. Those living alone were also excluded from the study, because the intervention included home assignments that should be completed with their families (mentioned below). Those who were diagnosed as medically (e.g. heart disease) or physically (e.g. osteoarthritis) contraindicated for PA were excluded from the PA group.

Of the 295 subjects assessed as having a CDR of 0.5, 127 (52 males and 75 females) fulfilled the inclusion criteria and agreed to participate in the study. Written informed consent was obtained from each participant. These 127 participants were divided into 18 clusters based on their place of living; the size of each cluster was 6-9 persons. Each cluster was randomly assigned to one of three groups (CI, PA or GRA), each consisting of 6 clusters. The number of subjects in the CI, PA and GRA groups was 45, 38 and 44, respectively (fig. 1).

\section{Assessment Measures}

MMSE, word fluency (WF), Trail Making Test part A (TMT-A) [19], 6-meter walk time and Quality of Life (QOL) Face Scale scores were measured as primary outcomes. The MMSE was selected as a measurement of general cognitive functions, and WF and the TMT-A were chosen as measures of executive functions. Physical ability was evaluated by 6-meter walk time. For the 6-meter walk, two trials were performed, and the mean of the two trials was used for the analysis. Subjective well-being was evaluated by the QOL Face Scale. This is a 9-choice picture format with a score range of $1-9$ (worst $=1$, best $=9$, from frowning to smiling faces) assessing the degree of general happiness in current daily life. The Geriatric Depression Scale (GDS) short version [20] was used to evaluate and screen depressive states. Two well-trained raters (Y.T. and M.N.) were responsible for the neuropsychological tests (MMSE, WF and TMT-A). Both pre- and postintervention assessments of the neuropsychological tests (MMSE, WF and TMT-A) as well as the 6-meter walk time for each participant were measured by the same raters. However, these raters were not blinded to the intervention type assigned to each subject.

To clarify the effect of the 'enjoyableness' of an intervention on the outcome, an analysis of the patient-reported outcome (PRO) was performed. For the purpose of avoiding any potential bias originating from a situation in which the methodology was overlapping with the evaluation of QOL, we chose not to ask the participants to rate the experience. The procedure of the PRO evaluation was as follows:

1 After having completed all the sessions, the participants were asked to write short essays that expressed their impressions, opinions, experiences, etc. concerning the interventions.

2 Two raters who were blinded to the identity of the writers read and evaluated the essays. The evaluation focused on the attitudes and emotions expressed in the texts. If the overall emotional expression of an essay was positive, the rater classified it as 'enjoyable', otherwise (if the emotional expression was negative or neutral) 'not enjoyable'.

3 If the raters disagreed on their rating, an agreement was achieved based on discussions.

\section{Interventions}

Each cluster of 6-9 persons (as mentioned above) received one of the three interventions (CI, PA or GRA) that was assigned to the cluster. All of them consisted of 12 group sessions and 12 home assignments. One instructor and 2 assistants attended each session of each cluster. Each group session was held once per week and contained an intervention program 
Nakatsuka et al.: A Cluster Randomized Controlled Trial of Nonpharmacological

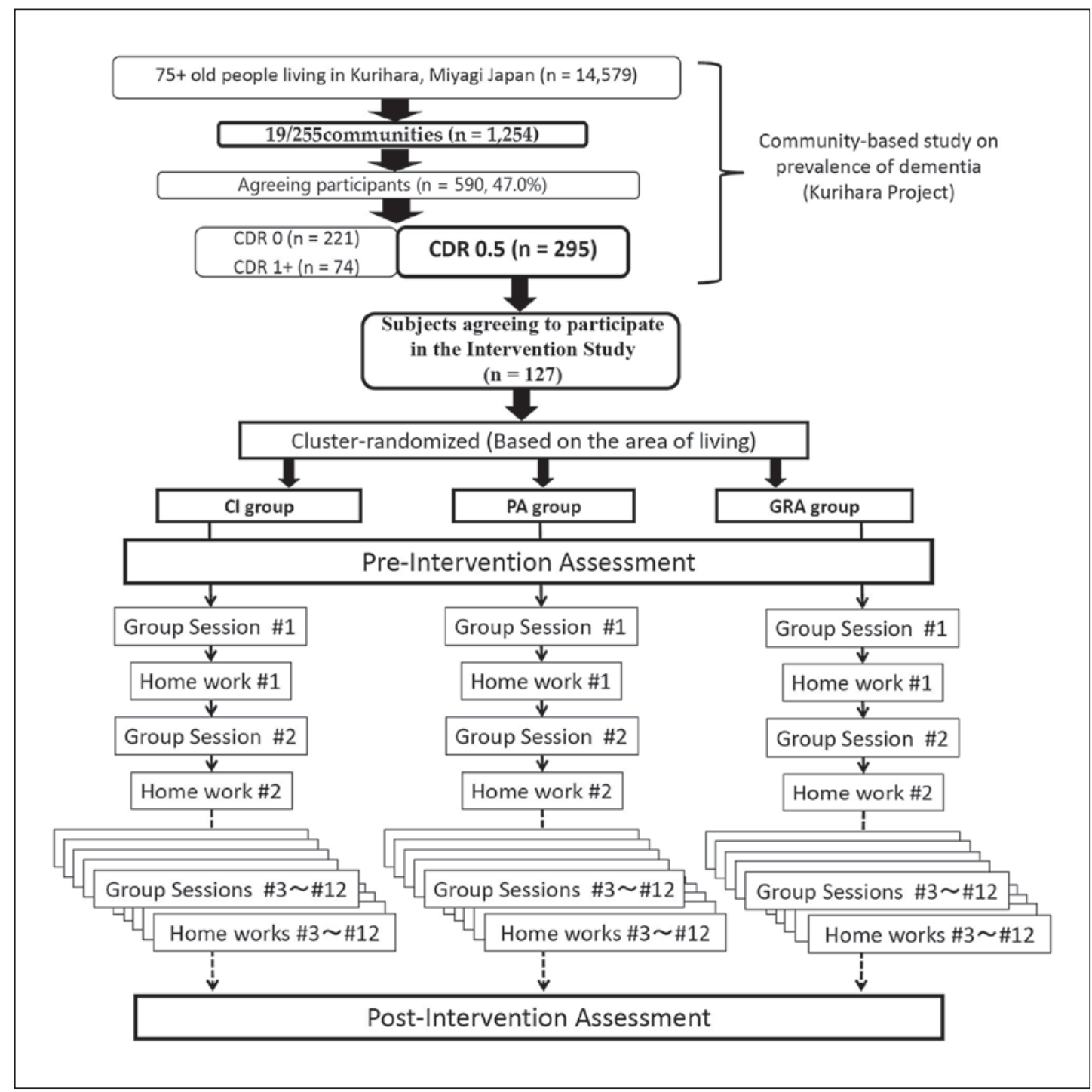

Fig. 1. Procedure of subject recruitment. 295 out of 590 individuals were assessed with a CDR of 0.5 (MCI) in the Kurihara Project, a community-based study on dementia; 127 of the 295 people with MCI who provided informed consent were enrolled in the study. These participants were cluster randomized and assigned to three intervention groups: CI, PA or GRA. Each series of interventions consisted of 12 sessions and 12 home assignments ('home work'). Each session was held once per week.

(60 min in total) that was interrupted by a tea break ( $5 \mathrm{~min}$ ). Instructions about the tasks for the home assignment were given at the end of each group session ( $5 \mathrm{~min}$ ). The home assignments were designed according to the intervention methods and contained tasks that require about $1 \mathrm{~h}$ to be completed. Written instructions were also given to the participants as an aid to successfully complete the home assignments. The group sessions were held in public halls or community centers of the regions of living of the participants. The home assignments were instructed to be done with the families of the participants (fig. 1).

For the $\mathrm{CI}$, the timetable of each session contained a series of questions, puzzles and games ( $60 \mathrm{~min}$ in total) that was interrupted by a tea break ( $5 \mathrm{~min}$ ). Instructions about the tasks for the home assignments were given at the end of each group session ( $5 \mathrm{~min}$ ). Efforts 
were made to entertain the subjects as well as to elicit the best individual performance within a flexible framework. The questions, puzzles and games targeted cognitive domains especially of executive function and attention. The tasks included WF games, crossword puzzles, anagram solving, spotting the difference between two pictures, maze solving, playing cards such as 'Old Maid', calculations and sudoku (number place). The categories of words given in the WF games avoided the ones that appeared in the pre- and postintervention assessments. To avoid any potential overlap with the interventions of the PA and/or GRA groups, tasks that might have provoked remote memory or required PA were minimized.

For the PA, the timetable of each session contained a series of exercises ( $60 \mathrm{~min}$ in total) that was interrupted by a tea break ( $5 \mathrm{~min}$ ). Instructions about the tasks for the home assignment were given at the end of each group session $(5 \mathrm{~min})$. Each session and home assignment consisted of 6 sets of exercises of 3-5 min of duration that were done every 10 min. The exercises were composed of walking and step aerobics using STEPWELL 2 (Konami Sports \& Life Co. Ltd., Tokyo, Japan). The intensity of each set of exercise was adjusted to '13 (slightly hard)', '14' or '15 (moderately hard)' according to the Borg Rating of Perceived Exertion (RPE) [21]. This rating was assigned by each participant for each set of exercise. The RPE is a scale to document the subjective exertion, and it may be used for prescribing and monitoring exercise intensity. Although this is a subjective measure, a person's exertion rating may provide a fairly good estimate of biological parameters including the actual heart rate $[22,23]$. The RPE is especially useful in practice or clinical settings, because self-monitoring how hard one's body is working may easily allow for self-adjustment of the intensity of activity. To assign the Borg RPE, the subjects selected one of the scores ranging from 6 to 20 , where 6 and 20 correspond to 'no exertion at all' and 'maximum exertion', respectively.

For the GRA, each session and home assignment consisted of talks about the current days and events of daily life (reality orientation) and discussions about memories of earlier days (reminiscence approach). The timetable of each session contained a reality orientation part (10 $\mathrm{min}$ ) and a set of reminiscence approach parts (50 $\mathrm{min}$ in total) that were interrupted by a tea break (5 min). Instructions for the home assignments were given at the end of each group session ( $5 \mathrm{~min}$ ). All sessions were hosted and the participants were encouraged by the instructor. The instructor presented a topic for each reminiscence approach part. Topics included activities, jobs, events and experiences from earlier days including childhood and adolescence. Pictures or items that related to these topics were also presented as an aid to discussion.

\section{Statistical Analyses}

Analyses were performed for the subjects who completed the protocol (protocol completion analysis). The effect of the cluster randomization was not accounted for in the statistical analysis. PASW statistics 18 software (SPSS Inc., Chicago, Ill., USA) was used for all statistical analyses.

Paired $t$ tests were performed for the pre- and postintervention values of all assessment measures for all participants. For these analyses, the significance level was set at $p<0.005$, uncorrected for multiple comparisons. Then, repeated-measures analysis of variance (ANOVA) was performed for each assessment measure to test the between-group difference regarding the intervention type. The significance level for the interaction between intervention type and each assessment measure was set to $\mathrm{p}<0.05$, uncorrected for multiple comparisons. If the between-group difference was significant, post hoc paired tests were performed for the pre- and postintervention values for each intervention method. The significance level was set at $\mathrm{p}<0.01$, uncorrected for multiple comparisons.

Repeated-measures ANOVA was performed for each assessment measure to test the between-group difference in PRO conditions ('enjoyable' and 'not enjoyable'). The signifi- 


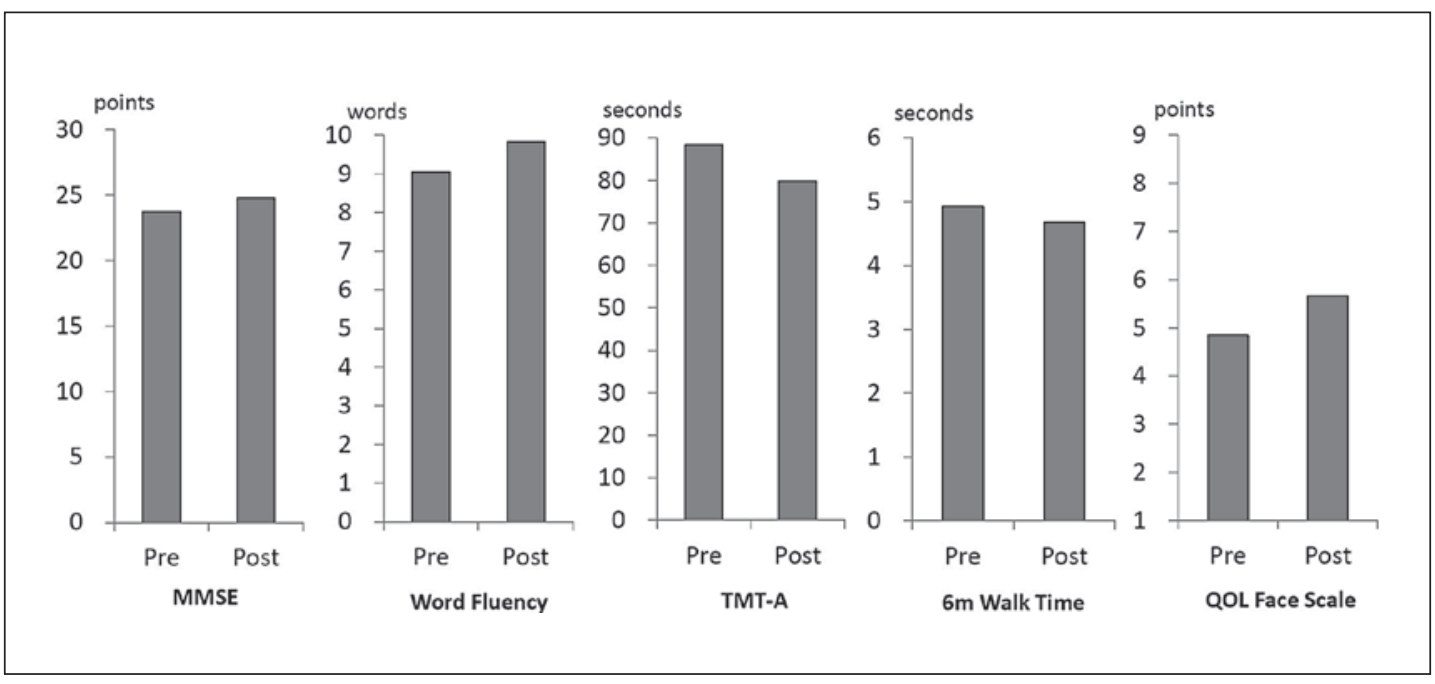

Fig. 2. Pre- and postintervention mean values of outcome measures for all participants. The MMSE, WF, TMTA, 6-meter $(6 \mathrm{~m})$ walk time and QOL Face Scale values of all participants improved significantly $(\mathrm{p}<0.005$, uncorrected for multiple comparisons).

cance level was set at $\mathrm{p}<0.01$, uncorrected for multiple comparisons. If the between-group difference in PRO conditions was significant, post hoc paired $t$ tests were performed for the pre- and postintervention values of each PRO condition. The significance level was set at $\mathrm{p}<$ 0.01 , uncorrected for multiple comparisons. Then the interaction between PRO condition and intervention type was also evaluated using repeated-measures ANOVA. The significance level was set at $\mathrm{p}<0.05$, uncorrected for multiple comparisons.

Responder analyses were made for each primary outcome. The cutoff points for the MMSE, WF, TMT-A, 6-meter walk time and QOL Face Scale were set to 2 points, 2 words, $2 \mathrm{~s}$, $0.01 \mathrm{~s}$ and 2 points, respectively. For each outcome measure, responders were defined as subjects who showed improvement that was equal to or greater than the cutoff point. Mean ages, mean years of education, GDS scores and baseline values of all the primary outcomes (MMSE, WF, TMT-A, 6-meter walk time and QOL Face Scale) were compared between responders and nonresponders. Effects of sex and type of intervention were also evaluated. Student's t test was used to compare mean ages, mean years of education, GDS scores and baseline values of the primary outcomes. The significance level was set at $p<0.01$, uncorrected for multiple comparisons. The effect of sex and intervention type was evaluated using the $\chi^{2}$ test. The significance level was set at $\mathrm{p}<0.01$ as well, uncorrected for multiple comparisons.

\section{Results}

Of the 127 participants, 95 completed the protocol (74.8\%). The preintervention mean values $( \pm$ SD) for the MMSE, WF, TMT-A, 6-meter walk time, GDS and QOL Face Scale were 23.7 \pm 3.1 points, $9.1 \pm 3.1$ words $/ \mathrm{min}, 88.3 \pm 38.0 \mathrm{~s}, 4.9 \pm 1.3 \mathrm{~s}, 4.8 \pm 3.4$ points and $4.9 \pm 1.7$ points, respectively. The postintervention mean values for the respective assessment measures were $24.8 \pm 2.8$ points, $9.8 \pm 3.3$ words $/ \mathrm{min}, 79.9 \pm 35.6 \mathrm{~s}, 4.7 \pm 1.1 \mathrm{~s}, 4.5 \pm 3.1$ points and $5.6 \pm 1.8$ points. Improvements in MMSE, WF, TMT-A, QOL Face Scale and 6-meter walk time values were significant ( $\mathrm{p}<0.005$, uncorrected for multiple comparisons) (fig. 2). 
Table 1. Demographic data and preintervention assessment values for the three intervention groups

\begin{tabular}{lcccl}
\hline & CI & PA & GRA & $\begin{array}{l}\text { Difference } \\
\text { between groups }\end{array}$ \\
& & & & \\
Number of participants & 45 & 38 & 44 & \\
Number of subjects completing the protocol & 32 & 24 & 39 & \\
Subjects completing the protocol, \% & 71.1 & 63.2 & 88.6 & 0.021 , uncorrected \\
Male, \% & 53.1 & 33.3 & 51.3 & n.s. \\
Age, years & $82.2 \pm 3.8$ & $81.3 \pm 3.8$ & $81.2 \pm 4.0$ & n.s. \\
Education, years & $8.1 \pm 1.1$ & $8.8 \pm 1.5$ & $9.4 \pm 1.9$ & $<0.01$, uncorrected \\
MMSE score & $22.2 \pm 3.2$ & $23.5 \pm 2.4$ & $25.1 \pm 2.7$ & $<0.01$, uncorrected \\
WF, words/min & $8.7 \pm 2.9$ & $8.1 \pm 2.9$ & $10.0 \pm 3.1$ & n.s. \\
TMT-A, s & $95.8 \pm 40.2$ & $91.4 \pm 40.2$ & $80.3 \pm 33.8$ & n.s. \\
GDS score & $5.1 \pm 3.5$ & $3.5 \pm 2.7$ & $5.3 \pm 3.7$ & n.s. \\
6-meter walk time, s & $4.9 \pm 1.2$ & $5.1 \pm 1.3$ & $4.8 \pm 1.4$ & n.s. \\
QOL Face Scale score & $5.3 \pm 1.9$ & $4.6 \pm 1.5$ & $4.7 \pm 1.7$ & n.s. \\
GDS score & $5.1 \pm 3.5$ & $3.5 \pm 2.7$ & $5.3 \pm 3.7$ & n.s. \\
\hline
\end{tabular}

Values are presented as means \pm SD unless specified otherwise. ANOVA showed significant differences in mean years of education and preintervention values of the MMSE between the groups $(\mathrm{p}<0.01$, uncorrected for multiple comparisons). The completion rate of the PA group was relatively low (63.2\%), which may be accounted for by the generally limited physical tolerance of old people. n.s. = Not significant.

Table 2. Pre- and postintervention mean values $( \pm S D)$ of assessment measures for each intervention group

\begin{tabular}{|c|c|c|c|c|c|c|}
\hline & \multicolumn{2}{|c|}{ MMSE score } & \multicolumn{2}{|c|}{ WF, words/min } & \multicolumn{2}{|l|}{ TMT-A, s } \\
\hline & pre & post & pre & post & pre & post \\
\hline CI & $22.2 \pm 3.2$ & $24.5 \pm 3.4$ & $8.7 \pm 2.9$ & $9.3 \pm 3.1$ & $95.8 \pm 40.2$ & $85.8 \pm 39.1$ \\
\hline PA & $23.5 \pm 2.4$ & $24.2 \pm 2.2$ & $8.1 \pm 2.9$ & $9.1 \pm 3.3$ & $91.4 \pm 40.2$ & $86.3 \pm 38.7$ \\
\hline \multirow[t]{3}{*}{ GRA } & $25.1 \pm 2.7$ & $25.4 \pm 2.5$ & $10 \pm 3.1$ & $10.8 \pm 3.3$ & $80.3 \pm 33.8$ & $71 \pm 29.2$ \\
\hline & \multicolumn{2}{|l|}{ GDS score } & \multicolumn{2}{|c|}{ QOL Face Scale score } & \multicolumn{2}{|c|}{ 6-meter walk time, s } \\
\hline & pre & post & pre & post & pre & post \\
\hline CI & $5.1 \pm 3.5$ & $5 \pm 2.5$ & $5.3 \pm 1.9$ & $5.6 \pm 1.9$ & $4.9 \pm 1.2$ & $4.7 \pm 0.9$ \\
\hline PA & $3.5 \pm 2.7$ & $3.2 \pm 2.5$ & $4.6 \pm 1.5$ & $5.8 \pm 1.7$ & $5.1 \pm 1.3$ & $4.6 \pm 1.2$ \\
\hline GRA & $5.3 \pm 3.7$ & $4.9 \pm 3.7$ & $4.7 \pm 1.7$ & $5.7 \pm 1.7$ & $4.8 \pm 1.4$ & $4.7 \pm 1.3$ \\
\hline
\end{tabular}

Thirty-two out of 45 (71.1\%), 23 out of 38 (60.5\%) and 38 out of 44 (86.4\%) participants of the CI, PA and GRA groups, respectively, completed the protocol. Table 1 shows the demographic data and preintervention assessment values of the three groups. Repeated-measures ANOVA revealed that the between-group difference regarding the intervention type was significant for years of education (d.f. $=2, \mathrm{~F}=5.81, \mathrm{p}=0.004$ ) and MMSE score (d.f. $=2, \mathrm{~F}=$ $6.42, \mathrm{p}=0.002)$. Post hoc paired t tests revealed a significant improvement in MMSE score and 6-meter walk time only for the $\mathrm{CI}$ and the PA group, respectively ( $\mathrm{p}<0.01$, uncorrected for multiple comparisons). Table 2 and figure 3 show the pre- and postintervention mean values of the assessment measures for each intervention method.

The between-group difference in PRO conditions was significant for QOL Face Scale score (d.f. $=1, F=7.61, p=0.007$ ). The interaction with intervention type was not significant. The 


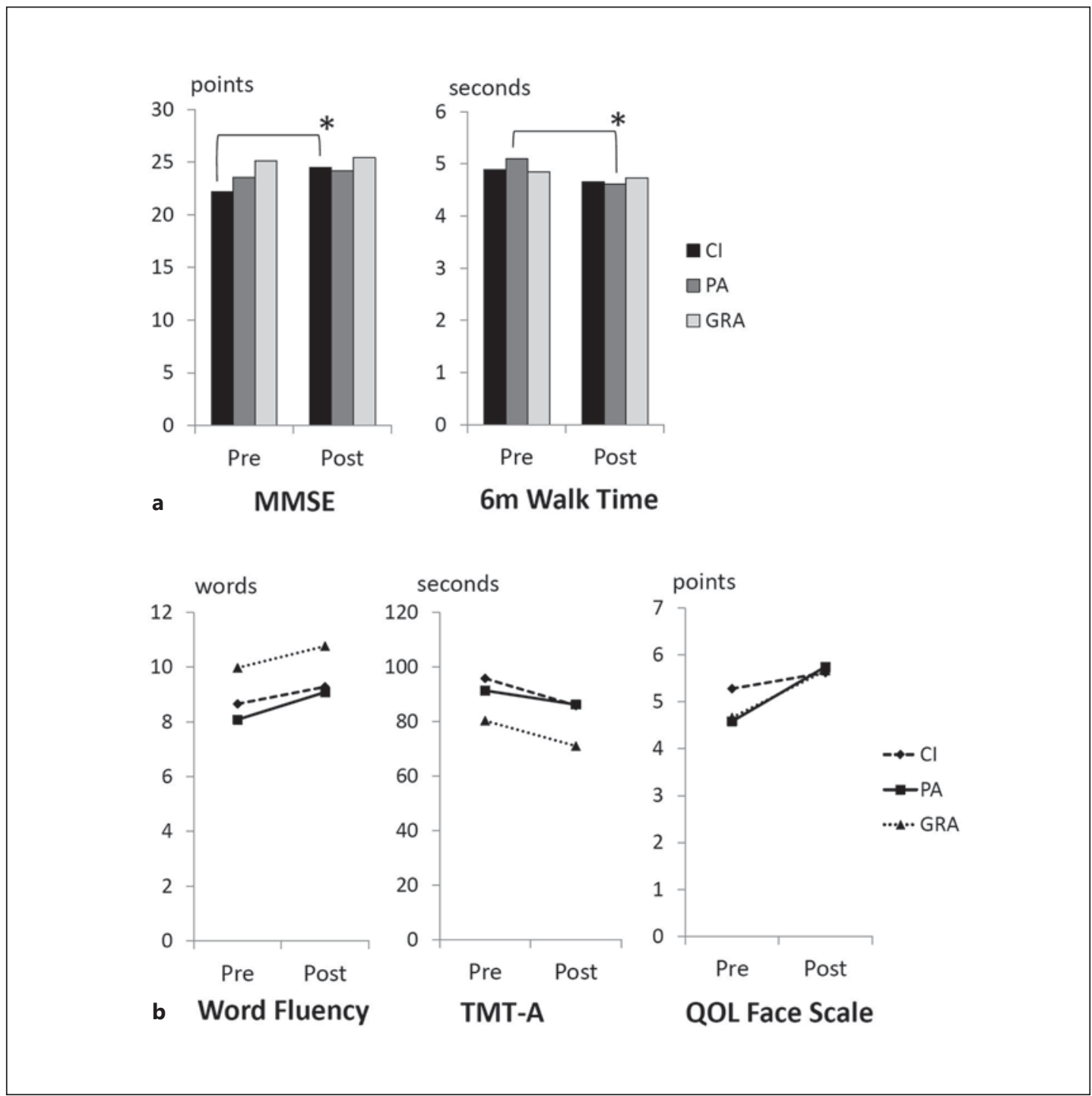

Fig. 3. Pre- and postintervention mean values of the MMSE, 6-meter (6m) walk time, WF, TMT-A and QOL Face Scale for the CI, PA and GRA groups. Repeated-measures ANOVA revealed a significant effect of intervention type for the MMSE and 6-meter walk time ( $p<0.05$, uncorrected for multiple comparisons). The post hoc paired t test revealed a significant improvement in MMSE score for the CI group and in 6-meter walk time for the PA group $(* \mathrm{p}<0.01$, uncorrected for multiple comparisons; a). No effect of the intervention type was found for WF, TMT-A and QOL Face Scale (b).

post hoc paired t test showed a significant improvement in QOL Face Scale scores in the 'enjoyable' group ( $\mathrm{p}<0.01$, uncorrected for multiple comparisons) (fig. 4).

Responders to the MMSE showed a significantly lower mean baseline MMSE score. The scores for the responders and nonresponders were $22.4 \pm 2.5$ and $24.8 \pm 3.0$, respectively. Responders to the QOL Face Scale showed significantly more years of education. The mean years of education of the responders and nonresponders were $9.4 \pm 1.9$ and $8.4 \pm 1.4$, respectively. None of the other parameters showed any significant difference between responders and nonresponders. The numbers of responders and nonresponders for each outcome as well as the statistically significant baseline parameters are shown in table 3 . 
Fig. 4. Pre- and postintervention mean values of the QOL Face Scale for the 'enjoyable' and 'not enjoyable' groups. Repeated-measures ANOVA revealed a significant effect of PRO without significant interactions with intervention type ( $p<0.05$, uncorrected for multiple comparisons). The post hoc paired t test showed a significant improvement in QOL Face Scale scores in the 'enjoyable' group ${ }^{*} \mathrm{p}<0.01$, uncorrected for multiple comparisons). ns = Not significant.

(C) 2015 S. Karger AG, Basel www.karger.com/dee

Nakatsuka et al.: A Cluster Randomized Controlled Trial of Nonpharmacological Interventions for Old-Old Subjects with a CDR of 0.5: The Kurihara Project

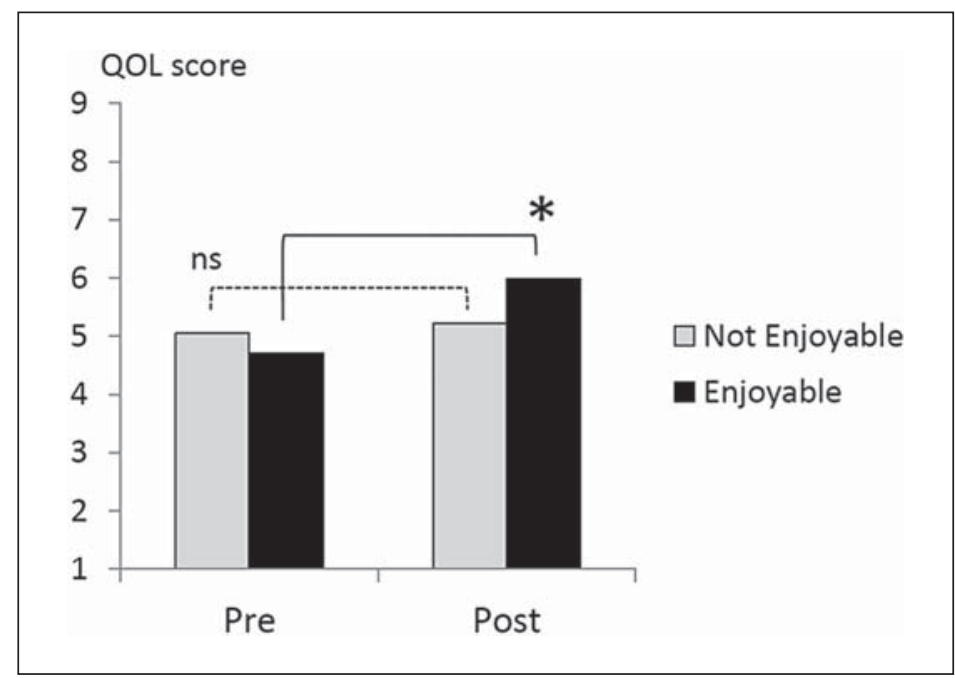

Table 3. Numbers of responders and nonresponders for each primary outcome and statistically significant baseline parameters (responder analysis)

\begin{tabular}{lccc}
\hline & Responders & Nonresponders & $\mathrm{p}$ \\
\hline $\begin{array}{l}\text { MMSE, } \mathrm{n} \\
\text { Intervention type, } \mathrm{n}\end{array}$ & 42 & 53 & \\
$\quad$ CI & 21 & 11 & 0.011 , uncorrected \\
PA & 8 & 16 & \\
GRA & 13 & 26 & $<0.001$, uncorrected \\
MMSE score & $22.4 \pm 2.5$ & $24.8 \pm 3.0$ & $<0.005$, uncorrected \\
\hline QOL, n & 35 & 60 & \\
Education, years & $9.4 \pm 1.9$ & $8.4 \pm 1.4$ & \\
\hline WF, n & 36 & 59 & \\
\hline TMT-A, n & 35 & 60 & \\
\hline 6-meter walk, $\mathrm{n}$ & 33 & 62 & \\
\hline
\end{tabular}

Mean ages, mean years of education, mean scores of the GDS and baseline values of all primary outcomes (MMSE, WF, TMT-A, 6-meter walk time and QOL Face Scale) were compared between the responders and nonresponders. For each outcome measure, responders were defined as subjects who showed improvement that was equal to or greater than the cutoff point.

Better improvement in MMSE and QOL Face Scale scores correlated with lower baseline MMSE score $(p<0.001)$ and more years of education $(p<0.005)$, respectively. A weak but nonsignificant effect of the type of intervention was found for the outcome of the MMSE ( $p=0.011)$.

\section{Discussion}

The present study clarified the effects related to three popular nonpharmacological interventions (CI, PA and GRA) based on a cluster RCT design. According to our results, CI may improve cognitive functions as evaluated by the MMSE in elderly subjects who were classified with a CDR of 0.5 , while PA may have a positive effect on physical ability. Executive functions as evaluated by WF and the TMT-A as well as subjective well-being as evaluated by the QOL 
Face Scale may improve irrespective of the three types of intervention. 'Enjoyableness' may be a key factor for the improvement in subjective well-being regardless of the intervention type. According to the responder analysis, a lower baseline MMSE score may predict a better outcome on the MMSE, while a higher educational level of a subject may be a predictor of greater improvement in QOL. The mean baseline MMSE score of our subjects was relatively low (23.7), which may be a consequence of their relatively few years of education. The result of WF was also low. Although people with MCI may show lower scores of WF than normal subjects [24], the scores may also be accounted for by the low educational level of our subjects [25].

According to previous studies, cognitive training may moderately improve cognitive functions [5, 7]. However, the evidence for this effect remained weak because of a lack of active controls. Our study compared the effects of cognitive training with two other active conditions, PA and GRA, which enabled evaluations of condition-specific as well as noncondition-specific effects of the interventions. Our findings suggest that the intervention that contains cognitive trainings may add to the improvements in cognitive functions that may occur as a consequence of the nonspecific effects of the interventions.

Previous studies have suggested that exercise may be one of the promising strategies for improving cognitive functions. Resistance [26,27] as well as aerobic trainings [28] may positively impact cognitive functioning and result in functional plasticity in healthy older adults. Furthermore, exercise training may have cognitive benefits for seniors with MCI [4, 29], especially improvements in selective attention and conflict resolution, processing speed and verbal fluency in senior women with amnestic MCI [4]. Thus, many previous studies have emphasized the positive impact of PA on the executive functions of subjects in the boundary state between normal aging and dementia. Indeed, our results support these previous findings; however, they also show that the benefit for executive functions may be a nonspecific effect that may occur with CI or the GRA as well. Nevertheless, we cannot exclude the secondary benefit of PA on cognitive functions, because an improvement in physical ability may potentially ameliorate cognition in the course of subsequent daily life.

The GRA has been shown to be beneficial to psychosocial well-being as well as to some cognitive abilities such as orientation and remote memory in demented patients $[8,13,15]$. Our findings are compatible with those of previous studies; however, they suggest that the improvements in subjective QOL and executive function may not be specific effects of the GRA.

Additionally, we evaluated the effect of PRO on the outcome measures. While a significant correlation with cognitive functions was not found, our results showed that better attitudes to or impressions of the experience with the interventions were associated with greater improvement in subjective QOL without an effect of the type of methodology. We previously reported that better PRO was associated with greater improvement in Multidimensional Observation Scale for Elderly Subjects (MOSES) scores in subjects participating in the GRA [30]. Our results support and extend the previous report, showing that 'enjoyableness' may be one of the key factors that may be associated with improvement in subjective well-being regardless of which methodology of nonpharmacological intervention is employed.

According to the responder analysis, a lower baseline MMSE score and a higher educational level of a subject may predict greater improvement in MMSE scores and QOL, respectively. Since the CDR evaluation mainly focuses on the level of independence in real life, this finding suggests that people with lower scores on the MMSE with a relatively preserved capacity of daily living may be better candidates for nonpharmacological interventions, especially CI. The biological, psychological or social background of the favorable effect of a higher educational level on improvement in QOL may deserve further investigation.

A limitation of the present study is that the ANOVA of the preintervention MMSE scores showed a significant difference in mean values between the groups. Since we did not rear- 
range the group assignment of the subjects in order to adjust for MMSE scores, it is difficult to exclude any bias derived from the phenomenon of 'regression to the mean', which might act in favor of the outcome of the CI group. Another limitation is that the dropout ratios were not similar between the groups; especially that for the PA group was high. This might be accounted for by the relatively limited physical capacity of old-old people. Other limitations include a lack of negative controls and data on long-term effects of the interventions. With regard to the lack of negative controls, we considered that there is a scientifically essential problem concerning the definition of 'placebo' with regard to nonpharmacological interventions, because it is actually impossible to hide from the subjects what kind of interventions they are participating in. From this point of view, the 'placebo' of a nonpharmacological intervention may be a paradox. We selected a period of 12 weeks between assessments, although this may be relatively short as compared with the periods used in other studies, which may range from 4 weeks to 1 year or more [3-10]. Indeed, our methodology might have limited an evaluation of long-term outcomes of the interventions. However, we considered that an intervention period of 12 weeks might be optimal and acceptable in order to successfully detect both the intervention-related and methodology-specific short-term effects from a statistical point of view. One of the findings that support the use of a period of 12 weeks between assessments has been made in a previous study showing the effect of a pharmacological intervention with donepezil on Alzheimer's disease [31]: the cognitive functions of a group medicated with $5 \mathrm{mg}$ of donepezil improved most greatly after 6-12 weeks, then declined and reverted to baseline levels 24 weeks after the introduction of medication.

In conclusion, our study clarified the short-term effects of nonpharmacological interventions. CI and PA may have specific benefits to cognitive functions and physical abilities, respectively. Executive functions and QOL may improve irrespective of the intervention type employed. The 'enjoyableness' of an intervention and a higher educational level of a subject may be key factors for improving QOL regardless of the intervention methodology. This is the first RCT of the GRA in subjects at the boundary of or in the transitional state between normal aging and dementia and a pilot trial directly comparing CI, PA and the GRA.

\section{References}

$\checkmark 1$ Lonie JA, Parra-Rodriguez MA, Tierney KM, Herrmann LL, Donaghey C, O'Carroll RE, Ebmeier KP: Predicting outcome in mild cognitive impairment: 4-year follow-up study. Br J Psychiatry 2010;197:135-140.

-2 Palmer K, Bäckman L, Winblad B, Fratiglioni L: Detection of Alzheimer's disease and dementia in the preclinical phase: population based cohort study. BMJ 2003;326:245.

-3 Cooper C, Li R, Lyketsos C, Livingston G: Treatment for mild cognitive impairment: systematic review. Br J Psychiatry 2013;203:255-264.

-4 Baker LD, Frank LL, Foster-Schubert K, Green PS, Wilkinson CW, McTiernan A, Plymate SR, Fishel MA, Watson GS, Cholerton BA, Duncan GE, Mehta PD, Craft S: Effects of aerobic exercise on mild cognitive impairment: a controlled trial. Arch Neurol 2010;67:71-79.

5 Gates NJ, Sachdev PS, Fiatarone Singh MA, Valenzuela M: Cognitive and memory training in adults at risk of dementia: a systematic review. BMC Geriatr 2011;11:55.

6 Ishizaki J, Meguro K, Ohe K, Kimura E, Tsuchiya E, Ishii H, Sekita Y, Yamadori A: Therapeutic psychosocial intervention for elderly subjects with very mild Alzheimer disease in a community: the Tajiri project. Alzheimer Dis Assoc Disord 2002;16:261-269.

7 Jean L, Bergeron ME, Thivierge S, Simard M: Cognitive intervention programs for individuals with mild cognitive impairment: systematic review of the literature. Am J Geriatr Psychiatry 2010;18:281-296.

-8 Lai CK, Chi I, Kayser-Jones J: A randomized controlled trial of a specific reminiscence approach to promote the well-being of nursing home residents with dementia. Int Psychogeriatr 2004;16:33-49.

$>9$ Nagamatsu LS, Handy TC, Hsu CL, Voss M, Liu-Ambrose T: Resistance training promotes cognitive and functional brain plasticity in seniors with probable mild cognitive impairment. Arch Intern Med 2012;172:666668.

$\$ 10$ Reijnders J, van Heugten C, van Boxtel M: Cognitive interventions in healthy older adults and people with mild cognitive impairment: a systematic review. Ageing Res Rev 2012;12:263-275. 
Nakatsuka et al.: A Cluster Randomized Controlled Trial of Nonpharmacological Interventions for Old-Old Subjects with a CDR of 0.5: The Kurihara Project

11

$>12$ review. Neurosci Biobehav Rev 2012;36:1163-1178.

13 Cotelli M, Manenti R, Zanetti O: Reminiscence therapy in dementia: a review. Maturitas 2012;72:203-205.

14 Norris A: Reminiscence with Elderly People. London, Winslow, 1986.

15 Hughes CP, Berg L, Danziger WL, Coben LA, Martin RL: A new clinical scale for the staging of dementia. Br J Psychiatry 1982;140:566-572.

16 Meguro K, Tanaka N, Kasai M, Nakamura K, Ishikawa H, Nakatsuka M, Satoh M, Ouchi Y: Prevalence of dementia and dementing diseases in the old-old population in Japan: the Kurihara Project. Implications for long-term care insurance data. Psychogeriatrics 2012;12:226-234.

17 Morris JC: The Clinical Dementia Rating (CDR): current version and scoring rules. Neurology 1993;43:24122414.

18 Folstein MF, Folstein SE, McHugh PR: 'Mini-mental state'. A practical method for grading the cognitive state of patients for the clinician. J Psychiatr Res 1975;12:189-198.

19 Lezak MD, Howieson DB, Loring DW: Neuropsychological Assessment, ed 4. New York, Oxford University Press, 2004.

20 Sheikh JI, Yesavage JA: Geriatric Depression Scale (GDS): Recent Evidence and Development of a Shorter Version. New York, Haworth, 1986.

21 Borg G: Perceived exertion as an indicator of somatic stress. Scand J Rehabil Med 1970;2:92-98.

-22 Dishman RK: Prescribing exercise intensity for healthy adults using perceived exertion. Med Sci Sports Exerc 1994;26:1087-1094.

23 Whaley MH, Woodall T, Kaminsky LA, Emmett JD: Reliability of perceived exertion during graded exercise testing in apparently healthy adults. J Cardiopulm Rehabil 1997;17:37-42.

24 Rinehardt E, Eichstaedt K, Schinka JA, Loewenstein DA, Mattingly M, Fils J, Duara R, Schoenberg MR: Verbal fluency patterns in mild cognitive impairment and Alzheimer's disease. Dement Geriatr Cogn Disord 2014;38: $1-9$.

-25 Tombaugh TN, Kozak J, Rees L: Normative data stratified by age and education for two measures of verbal fluency: FAS and Animal Naming. Arch Clin Neuropsychol 1999;14:167-177.

26 Liu-Ambrose T, Nagamatsu LS, Graf P, Beattie BL, Ashe MC, Handy TC: Resistance training and executive functions: a 12-month randomized controlled trial. Arch Intern Med 2010;170:170-178.

27 Liu-Ambrose T, Nagamatsu LS, Voss MW, Khan KM, Handy TC: Resistance training and functional plasticity of the aging brain: a 12-month randomized controlled trial. Neurobiol Aging 2011;33:1690-1698.

28 Colcombe SJ, Kramer AF, Erickson KI, Scalf P, McAuley E, Cohen NJ, Webb A, Jerome GJ, Marquez DX, Elavsky S: Cardiovascular fitness, cortical plasticity, and aging. Proc Natl Acad Sci USA 2004;101:3316-3321.

-29 Lautenschlager NT, Cox KL, Flicker L, Foster JK, van Bockxmeer FM, Xiao J, Greenop KR, Almeida OP: Effect of physical activity on cognitive function in older adults at risk for Alzheimer disease: a randomized trial. JAMA 2008;300:1027-1037.

-30 Meguro K, Akanuma K, Meguro M: Patient-reported outcome is important in psychosocial intervention for dementia: a secondary analysis of a randomized controlled trial of group reminiscence approach data. Dement Geriatr Cogn Disord Extra 2013;3:37-38.

-31 Burns A, Rossor M, Hecker J, Gauthier S, Petit H, Möller HJ, Rogers SL, Friedhoff LT: The effects of donepezil in Alzheimer's disease - results from a multinational trial. Dement Geriatr Cogn Disord 1999;10:237-244. 